\title{
Evolutionary stability in the generalized second-price auction
}

\section{Thomas W. L. Norman ${ }^{1}$}

Received: 12 March 2019 / Accepted: 29 November 2019 / Published online: 9 December 2019 (c) The Author(s) 2019

\begin{abstract}
The "generalized second-price auction" is widely employed to sell internet advertising positions and has many equilibria. Analysis of this auction has assumed that myopic players commonly know each others' position values, and that the resulting equilibrium play is "locally envy-free". Here, I argue that the appropriate refinement of Nash equilibrium for this setting is evolutionary stability, and show that it implies that an equilibrium is locally envy-free if the whole population of players bids in each auction and the set of possible bids is not too coarse. However, not all locally envy-free equilibria are evolutionarily stable in this case, as I show by example for the popular Vickrey-Clarke-Groves outcome. The existence of evolutionarily stable equilibrium is established when one position is auctioned, as well as for two positions and a large number of bidders.
\end{abstract}

Keywords Online advertising · Internet auctions · Position auctions · Evolutionarily stable strategy · Locally envy-free equilibrium - Symmetric Nash equilibrium .

Vickrey-Clarke-Groves outcome $\cdot$ Spite

JEL Classification C73 · D44 · L81 $\cdot$ L86 · M3

\section{Introduction}

The "generalized second-price auction" (GSP) is a popular mechanism for the sale of advertising slots, or "sponsored links", on internet search engines. Advertisers can bid on possible search keywords, for each of which there is such a "sponsored search" or "position" auction, taking place in continuous time. Higher positions are more valuable, in the sense that they generate more clicks, and the GSP allocates these positions in order of bid size, but with each advertiser's payment determined by the

Thomas W. L. Norman

thomas.norman@magd.ox.ac.uk

1 Magdalen College, Oxford, UK 
next highest bid. Notwithstanding the complexity of this repeated game of incomplete information, much of the existing literature has argued that it is well modelled as a one-shot game of complete information.

Whilst there exist many equilibria of even this one-shot game, much work has focused on Varian's (2007) "symmetric Nash equilibria", which are Nash equilibria in which bidders do not wish to deviate to win a higher position even if they only have to pay their current bid. Edelman et al. (2007) justify symmetric equilibria with their "locally envy-free" requirement - that a bidder will raise her bid to induce a higher payment for the bidder in the position above her, but only up to the point at which she would not regret her bid if that opponent were then to undercut her slightly. Börgers et al. (2013) "regard this argument as not entirely compelling because it is not clear that the relevant case for bidders to consider is the case that other bidders lower their bids" in this way. Moreover, it is not clear why a bidder should raise her bid to harm her opponent when there is no such motivation captured in her payoffs.

Unless, that is, the advertisers' bids are evolutionarily stable (Maynard Smith and Price 1973; Schaffer 1988). ${ }^{1}$ Roughly speaking, an evolutionarily stable strategy (ESS) is a strategy that, if adopted by most players, cannot be outperformedand hence invaded-by a small number of players using a "mutant" strategy. This concept was originally defined for infinite populations, where it refines Nash equilibrium, but was generalized to allow finitely many players by Schaffer (1988). In the infinite-population case, each game involves an arbitrarily small fraction of the population, which is an unrealistic assumption for sponsored search auctions. Indeed, implicit in the one-shot complete-information modelling of these games is the assumption that a fixed set of advertisers participate in each auction, in which case the whole population takes part in each game. Schaffer's analysis allows for this "playing the field" (Maynard Smith 1982) scenario, an important implication of which is that stable behaviour may be "spiteful" (Hamilton 1971): an ESS player acts not only to increase her payoff as usual, but also to decrease the payoffs of her opponents. This spite motivation, coupled with the robustness of ESS to small mutations, is key to my finding here that evolutionarily stable equilibrium bids in the "playing the field" GSP are locally envy-free (if the set of possible bids is not too coarse).

The potential role for spite in explaining bidding behaviour in auctions predates the literature on sponsored search: It has been offered as an explanation for the experimental regularity of overbidding in second-price auctions (Morgan et al. 2003), where there is evidence that bidders overbid more often when they are likely to lose and set the winner's price (Andreoni et al. 2007; Cooper and Fang 2008; Nishimura 2011). However, to my knowledge, evolutionary stability offers the first justification of such behaviour with standard preferences. In the infinite-population setting, ESS implies Nash equilibrium but need not exist in general. In the finite-population case, evolutionary stability may even diverge from Nash play (Ania 2008; Hehenkamp et al. 2010). However, I establish the existence of equilibrium ESS in the "playing the field" GSP

\footnotetext{
1 For general introductions to evolutionary game theory, see Weibull (1995), Sandholm (2010) or Newton (2018), for instance.
} 
for one position, and also for two positions as the number of bidders becomes large (which I argue extends to more than two positions). ${ }^{2}$

Whilst evolutionary stability can thus provide a foundation for locally envy-free equilibria, there remain many such equilibria, including one with the same prices as the truthful equilibrium of the Vickrey-Clarke-Groves (VCG) mechanism. This outcome has been a popular equilibrium selection, but its incomplete-information basis in Edelman et al. (2007) has been criticized for its arbitrary use of ascending bids (Börgers et al. 2013, p. 175, Che, Choi, and Kim 2007, p. 25). Cary et al. (2014) seek to justify the VCG outcome via a particular adaptive process, but their "balanced bidding" condition has been criticized by Che, Choi, and Kim (ibid.): "Since the same indifference condition is used to define the lower bound of symmetric Nash equilibria, and since the lower bound coincides with VCG, it is not surprising that the resulting dynamics converge to the VCG outcome". The same authors offer experimental evidence against the VCG outcome.

More traditional equilibrium refinements have been explored by Milgrom and Mollner (2018a), who find that whilst properness (Myerson 1978) and strategic stability (Kohlberg and Mertens 1986) are too weak to imply locally envy-free equilibrium in the GSP, it is implied by their pure "test-set equilibrium" refinement, and test-set equilibrium is equivalent to a modification of van Damme's (1984) quasi-perfect equilibrium in a high-stakes version of a finite game. However, pure test-set equilibrium does not imply the VCG outcome, which furthermore does not survive Hashimoto's (2013) "noise bidder" refinement, where a nonstrategic random bidder takes part in the auction with low probability. Here, I find similarly that evolutionary stability offers no support for the VCG outcome; I show by example that the VCG outcome need not be evolutionarily stable. Milgrom and Mollner (2018b), meanwhile, define an "extended proper equilibrium" - which is essentially a symmetric proper equilibrium of the symmetrized game employed by Selten (1980) and in my analysis below-and show that it too implies locally envy-free equilibrium in the GSP. In their paper and here, the symmetrizing of trembles/mutations across players is important for the result, and the relation between extended proper equilibrium and evolutionary stability would be an interesting topic for further study.

Evolutionary stability is a very natural refinement for sponsored search auctions. There are two elements to the one-shot complete-information simplification popular in the literature: first, that bidders' values are commonly known, because advertisers who "originally have private information about their types, gradually learn the values of others, and can adjust their bids repeatedly" (Edelman et al. 2007, p. 249); and second, that their payoffs are myopic, in the sense that their bids in any given auction are motivated by the immediate outcome of that auction rather than all future outcomes. It is well known that players can improve on myopic stage-game actions in repeated games, but Edelman, Ostrovsky, and Schwartz argue that they may not be able to do so in sponsored search auctions, due to the complexity of the requisite strategy implementation in a frequently repeated game: " bidding software must first be authorized

\footnotetext{
2 Alós-Ferrer and Ania (2001) define a weakening of Schaffer's ESS that allows only deviations to "nearby" strategies. This concept, which captures an appealing form of bounded rationality under learning, coincides with ESS for equilibrium strategies in the GSP.
} 
by the search engines, and search engines are unlikely to permit strategies that would allow advertisers to collude and substantially reduce revenues". 3

Given myopic behaviour, the common learning of bidders' valuations in an environment with frequent bidding is well founded theoretically (Jordan 1991) and experimentally (Che et al. 2017), and indeed, some platforms allow direct observation of competitor bids. Hence, the focus on Nash equilibria of one-shot sponsored search auctions appears valid, but the refinement possibilities offered by their repeated occurrence remain unexplored; for the framework of a game played repeatedly by myopic players is precisely that of evolutionary game theory, and the concept appropriate when the players are often the same advertisers is finite-population ESS. Edelman et al. (2007, p. 249) themselves ask "if the vector of bids stabilizes, at what bids can it stabilize?" This is a question of evolutionary game theory. In justifying their locally envy-free refinement, they ask "what are the simple strategies that an advertiser can use to increase his payoff, beyond simple best responses to the other players' bids?" This is a question of which mutants can successfully invade an otherwise stable vector of bids. Finally, they "view a locally envy-free equilibrium as a prediction regarding a rest point at which the vector of bids stabilizes" (ibid., p. 250). Evolutionary stability provides a basis for this view.

\section{Evolutionarily stable bids}

Consider a population of $M$ (risk-neutral) bidders repeatedly matched at random to play a $K$-player "generalized second-price" (GSP) auction for $N$ positions $i=$ $1, \ldots, N$, with $K \leq M$. In particular, the bidders simultaneously submit onedimensional bids $b_{k} \in\{0, \delta, 2 \delta, \ldots\} \equiv \mathcal{B}, \delta>0$, for payment per click. The winner $g(j)$ of position $j, j=1, \ldots, \min \{N, K\}$, is the bidder with the $j$ th highest bid $b^{(j)}$ (with ties split randomly), paying the $j+1$ th highest bid per click; any remaining bidders pay and receive nothing. The (expected) number of clicks per period received by the bidder in position $i$ is $\alpha_{i}$, so that bidder $g(i)$ 's total payment $p^{(i)}$ is $\alpha_{i} b^{(i+1)}$ for $i=1, \ldots, \min \{N, K\}$; if $N \geq K$, the last bidder's payment $p^{(K)}$ is equal to zero. Positions are labelled in descending order (without loss of generality): $i<j$ implies $\alpha_{i}>\alpha_{j}$. The value per click to bidder $k$ is $s_{k}$, with $S$ denoting the set of values $\left(s_{1}, s_{2}, \ldots, s_{K}\right)$; suppose that values are also labelled in descending order (again without loss of generality), and that no two players have the same value: $s_{1}>s_{2}>\cdots>s_{K} \cdot{ }^{4}$ Hence, bidder $k$ 's payoff from receiving position $i$ is

$$
\alpha_{i} s_{k}-p^{(i)}
$$

Each bidder $k$ chooses her bid myopically each period to maximize this payoff, without regard for subsequent payoffs. This setup follows that of Edelman et al. (2007); see

\footnotetext{
${ }^{3}$ However, Decarolis et al. (2017) explore the vulnerability of the GSP to collusive bidding through common marketing agencies.

${ }^{4}$ If multiple players shared the same value, then they would share the same equilibrium bid, in which case the position order $g$ would become stochastic and the definition of locally envy-free equilibrium below would require modification.
} 
Börgers et al. (2013, pp. 166-8) for discussion and relaxation of some of its limitations, such as the independence of value per click from position. Athey and Ellison (2011) endogenize players' values via an explicit model of consumer search.

Definition 1 (Edelman et al. 2007) An equilibrium of the simultaneous-move game induced by GSP is locally envy-free if a player cannot improve her payoff by exchanging bids with the player ranked one position above her; i.e. for any $i \leq \min \{N+1, K\}$,

$$
\alpha_{i} s_{g(i)}-p^{(i)} \geq \alpha_{i-1} s_{g(i)}-p^{(i-1)},
$$

where $\alpha_{N+1}=0$.

Under the VCG mechanism, by contrast, each advertiser pays the negative externality that her presence in the game imposes on the other players under truthful bids. The last advertiser allocated a position hence pays zero if $N \geq K$, and $\alpha_{N} b^{(N+1)}$ otherwise; for all other $i<\min \{N, K\}$, the payment induced by VCG is $p^{V,(i)}=\left(\alpha_{i}-\alpha_{i+1}\right) b^{(i+1)}+p^{V,(i+1)}$. If, in the GSP, $b^{(i)}=p^{V,(i-1)} / \alpha_{i-1}$ for each $i>1$ and $b^{(1)}=s_{1}$, then the bids constitute a locally envy-free equilibrium, with each advertiser's position and payment equal to those in the dominant-strategy equilibrium under the VCG mechanism (Edelman et al. 2007, Theorem 1); I will refer to this as the VCG outcome of the GSP.

Suppose that, prior to each auction, the values in the set $S$ are assigned (bijectively) at random to the players, with each possible assignment equally likely. A strategy $\sigma$ is a vector $\left(\sigma\left(s_{1}\right), \sigma\left(s_{2}\right), \ldots, \sigma\left(s_{K}\right)\right)$, specifying the bid $\sigma(s) \in \mathcal{B}$ that a bidder would make if she had each value $s \in S$. The strategy set is of course then symmetric, and a strategy profile $\left(\sigma_{1}, \sigma_{2}, \ldots, \sigma_{K}\right)$ implies a joint distribution $\mu$ over each bidder's value, position and total payment. There is then a symmetric payoff function $\pi\left(\sigma_{k} \mid \sigma_{-k}\right)=$ $\mathrm{E}_{\mu}\left(\alpha_{i} s_{k}-p^{(i)}\right)$ for each bidder $k$, where $\sigma_{-k}$ is (any permutation of) $\left(\sigma_{l}\right)_{l \neq k}$. This symmetrizing of the game is necessary for single-population ESS analysis and is the standard way of accommodating asymmetric games within this framework (Selten 1980). It is not intended to capture a genuinely incomplete-information setting (as in Edelman et al. 2007, p. 252, Athey and Nekipelov 2010, Gomes and Sweeney 2014, or Caragiannis et al. 2015). In particular, whilst players are ex ante uncertain of their values, there is common knowledge of the true set of values $S$. Since a player's evolutionarily stable strategy is symmetric and conditioned on her own realized value, whilst her payoffs are invariant to permutations in the bids of her opponents, this effectively remains the same game of complete information described earlier.

For example, consider a three-player GSP with values $S=\left\{s_{1}, s_{2}, s_{3}\right\}=\{9,5,2\}$. Prior to finding out their values, each of the three players chooses a strategy $\sigma=$ $(\sigma(9), \sigma(5), \sigma(2))$ that specifies the bid she would choose for each possible value. The values are then assigned to the players, with each assignment (e.g. $(9,5,2)$ or $(2,9,5))$ equally likely (and no two players sharing a value). Since ESS is a symmetric solution concept, there is no uncertainty in the game except that players do not know which bidder they are going to be. For example, when all players choose the strategy $\sigma=(\sigma(9), \sigma(5), \sigma(2))=(6,4,3)$, we always see the bids 6,4 , and 3 played by the bidders with values 9,5 , and 2 , respectively. 
I call $\sigma^{*}$ an equilibrium strategy if the bid vector $\left(\sigma^{*}(s)\right)_{s \in S}$ that it generates is a Nash equilibrium of the GSP. Given a strategy $\sigma$, let a mutant $\sigma_{m}$ be a strategy that differs from $\sigma$ in exactly one dimension (i.e. that makes a different bid to $\sigma$ for exactly one value); this definition ensures that a mutation in the symmetrized game corresponds to a single mutation/deviation in the original (asymmetric) GSP.

Definition 2 A strategy $\sigma_{\mathrm{ess}}$ is evolutionarily stable if, for all mutants $\sigma_{m}$,

$$
\begin{aligned}
(1 & \left.-\frac{K-1}{M-1}\right) \pi\left(\sigma_{\mathrm{ess}} \mid \sigma_{\mathrm{ess}}, \sigma_{\mathrm{ess}}, \ldots\right) \\
& +\frac{K-1}{M-1} \pi\left(\sigma_{\mathrm{ess}} \mid \sigma_{m}, \sigma_{\mathrm{ess}}, \ldots\right) \geq \pi\left(\sigma_{m} \mid \sigma_{\mathrm{ess}}, \sigma_{\mathrm{ess}}, \ldots\right)
\end{aligned}
$$

This is not the infinite-population definition of evolutionary stability (Maynard Smith and Price 1973; Maynard Smith 1982), but rather Schaffer's (1988) finite-population definition, where a single player "mutates" to $\sigma_{m} .{ }^{5}$ This mutant player may be matched to play a GSP against a given nonmutant player, who would then receive the expected payoff $\pi\left(\sigma_{\mathrm{ess}} \mid \sigma_{m}, \sigma_{\mathrm{ess}}, \ldots\right)$ from facing the mutant (in an unknown position). Alternatively, the nonmutant player may be matched exclusively with other nonmutants, thus receiving the expected payoff $\pi\left(\sigma_{\mathrm{ess}} \mid \sigma_{\mathrm{ess}}, \sigma_{\mathrm{ess}}, \ldots\right)$. In order to be evolutionarily stable, the overall expected payoff of the nonmutant must exceed that of the mutant (who cannot face himself), $\pi\left(\sigma_{m} \mid \sigma_{\mathrm{ess}}, \sigma_{\mathrm{ess}}, \ldots\right)$. In the GSP, we can think of ESS as capturing the bids of repeatedly and randomly matched advertisers, whose bids are stable in the face of one member of the advertiser population switching to another strategy.

Schaffer's concept of evolutionary stability includes the special "playing the field" case $K=M$, where the whole population takes part in each game, but also any instance where a proper subset of a finite population is matched to play the GSP. Notice that, unlike its infinite-population counterpart, symmetric play of a finite-population ESS need not be a Nash equilibrium (see, for example, Vega-Redondo 1996, Sect. 2.7). This relation is explored by Ania (2008) and Hehenkamp et al. (2010), who offer classes of games where finite-population ESS does refine Nash, but the GSP does not fit within any of these classes. Indeed, rather than Nash equilibrium, there seems to be a general relation between finite-population evolutionary stability and Walrasian equilibrium (Vega-Redondo 1997; Alós-Ferrer and Ania 2005). This relation finds a further instance in Proposition 2, in the sense that locally envy-free equilibrium can be interpreted as a Walrasian equilibrium: "If each bidder takes these prices as given and fixed, and picks the position that generates for him the largest surplus at these prices, then for each position there will be exactly one bidder who wants to acquire that position... [and] demand and supply are both equal to 1" (Börgers et al. 2013, p. 170).

\footnotetext{
5 In particular, Definition 2 is the first half of Schaffer's definition; I make no use of the stability condition in the second half of his definition.
} 


\subsection{Properties}

I begin by showing that positive evolutionarily stable bids differ by the minimum bid increment $\delta$, for $\delta$ sufficiently small. Intuitively, Schaffer's (1988) evolutionary stability leads players to behave "spitefully" towards one another, so that each will increase her bid to harm her opponents as long as her own payoff remains unaffected; thus, bids for consecutive positions cannot differ by more than $\delta$. To rule out tied bids, consider a situation in which bidders $k$ and $k+1$ make the same bid and $s_{k}>s_{k+1}$. Then either: (a) $s_{k}$ is considerably above $b_{k}$, in which case a profitable deviation exists (for sufficiently small $\delta$ ) whereby $k$ increases her bid and wins the higher position for sure; or (b) $s_{k}$ is very close to $b_{k}$, in which case $s_{k+1}$ must be less than $b_{k}=b_{k+1}$, so that a profitable deviation exists (for sufficiently small $\delta$ ) whereby bidder $k+1$ reduces his bid and avoids the risk of winning the position at that price.

Proposition 1 There exists $\bar{\delta}>0$ such that, for all $\delta<\bar{\delta}$, if $\sigma_{\mathrm{ess}}$ is an equilibrium evolutionarily stable strategy, then for all $i=1, \ldots, \min \{N, K-1\}, b_{\mathrm{ess}}^{(i)}=b_{\mathrm{ess}}^{(i+1)}+\delta$.

Proof Suppose otherwise; then for all $\delta>0$ there exists an equilibrium ESS $\sigma_{\text {ess }}$ and an $i \in\{1, \ldots, \min \{N, K\}\}$ such that $b_{\text {ess }}^{(i)} \neq b_{\text {ess }}^{(i+1)}+\delta$. But since $\sigma_{\text {ess }}$ is evolutionarily stable, it solves

$$
\sigma_{\mathrm{ess}} \in \underset{\sigma_{m}}{\arg \max } \pi\left(\sigma_{m} \mid \sigma_{\mathrm{ess}}, \sigma_{\mathrm{ess}}, \ldots\right)-\frac{K-1}{M-1} \pi\left(\sigma_{\mathrm{ess}} \mid \sigma_{m}, \sigma_{\mathrm{ess}}, \ldots\right)
$$

Since increasing bids without altering $g$ reduces the second term of the maximand at no cost to the first, $\sigma_{\text {ess }}$ implies a $b_{\text {ess }}^{(i)}$ (for $i=2, \ldots, \min \{N+1, K\}$ ) above which $g$ is altered with positive probability-i.e. bids for adjacent positions differ by at most $\delta$.

Furthermore, since $\sigma_{\text {ess }}$ is also an equilibrium strategy, I claim that no two players can make the same bid for $\delta>0$ sufficiently small. To see this, note that any player with a shared bid and a strictly negative expected payoff will wish to deviate to a zero bid. Hence, suppose that any player with a shared bid has a nonnegative expected payoff. But any player $k$ with the shared highest bid $b$ has a payoff at most

$$
\frac{1}{K} \alpha_{1}\left(s_{k}-b\right)+\frac{K-1}{K} \alpha_{j}\left(s_{k}-b+\delta\right)
$$

for some position $j \in\{2, \ldots, N+1\}$ (where $\alpha_{N+1}=0$ ), because there would be a profitable upwards deviation if $\alpha_{1}\left(s_{k}-b\right)$ strictly exceeded $\alpha_{j}\left(s_{k}-b+\delta\right)$ for all such $j$. This must hence weakly exceed her upward-deviation payoff $\alpha_{1}\left(s_{k}-b\right)$, which is true if and only if

$$
\begin{aligned}
\alpha_{j}\left(s_{k}-b+\delta\right) & \geq \alpha_{1}\left(s_{k}-b\right) \\
\Leftrightarrow \quad b & \geq s_{k}-\frac{\alpha_{j}}{\alpha_{1}-\alpha_{j}} \delta .
\end{aligned}
$$


Thus, letting $\bar{s}^{b}$ be the highest value and $\underline{s}^{b}$ the lowest value amongst the players sharing the bid $b$,

$$
b \geq \bar{s}^{b}-\frac{\alpha_{j}}{\alpha_{1}-\alpha_{j}} \delta
$$

This is inconsistent with nonnegativity of the expected payoff of the player with the lowest value $\underline{s}^{b}$ for

$$
\begin{aligned}
\frac{1}{K} \alpha_{1}\left(\underline{s}^{b}\right. & -b)+\frac{K-1}{K} \alpha_{j}\left(\underline{s}^{b}-b+\delta\right)<0 \\
& \Leftrightarrow b>\underline{s}^{b}+\frac{(K-1) \alpha_{j}}{\alpha_{1}+(K-1) \alpha_{j}} \delta \\
& \Leftarrow \bar{s}^{b}-\frac{\alpha_{j}}{\alpha_{1}-\alpha_{j}} \delta>\underline{s}^{b}+\frac{(K-1) \alpha_{j}}{\alpha_{1}+(K-1) \alpha_{j}} \delta \\
& \Leftrightarrow \delta<\frac{\left(\alpha_{1}-\alpha_{j}\right)\left(\alpha_{1}+(K-1) \alpha_{j}\right)}{K \alpha_{1} \alpha_{j}}\left(\bar{s}^{b}-\underline{s}^{b}\right) .
\end{aligned}
$$

Moreover, any player $k$ with the shared second highest bid of $b^{\prime}>0$ has a payoff at most

$$
\theta \alpha_{2}\left(s_{k}-b^{\prime}\right)+(1-\theta) \alpha_{j^{\prime}}\left(s_{k}-b^{\prime}+\delta\right)
$$

for some $\theta \in[0,1]$ and some position $j^{\prime} \in\{3, \ldots, N+1\}$. This must hence exceed her payoff $\left(\alpha_{1}\left(s_{k}-b^{\prime}-\delta\right)+\alpha_{2}\left(s_{k}-b^{\prime}\right)\right) / 2$ from deviating to $b^{\prime}+\delta$, which is true if and only if

$$
b^{\prime} \geq s_{k}-\frac{\alpha_{1}+2(1-\theta) \alpha_{j^{\prime}}}{\alpha_{1}+(1-2 \theta) \alpha_{2}-2(1-\theta) \alpha_{j^{\prime}}} \delta .
$$

Thus, letting $\bar{s}^{b^{\prime}}$ be the highest value and $\underline{s}^{b^{\prime}}$ the lowest value amongst the players sharing the bid $b^{\prime}$,

$$
b^{\prime} \geq \bar{s}^{b^{\prime}}-\frac{\alpha_{1}+2(1-\theta) \alpha_{j^{\prime}}}{\alpha_{1}+(1-2 \theta) \alpha_{2}-2(1-\theta) \alpha_{j^{\prime}}} \delta .
$$

This is inconsistent with nonnegativity of the expected payoff of the player with the lowest value $\underline{s}^{b^{\prime}}$ for

$$
\delta<\frac{\left(\theta \alpha_{2}+(1-\theta) \alpha_{j^{\prime}}\right)\left(\alpha_{1}+(1-2 \theta) \alpha_{2}-2(1-\theta) \alpha_{j^{\prime}}\right)}{2(1-\theta) \alpha_{1} \alpha_{j^{\prime}}+(1-\theta) \alpha_{2} \alpha_{j^{\prime}}+\theta \alpha_{1} \alpha_{2}}\left(\bar{s}^{b^{\prime}}-\underline{s}^{b^{\prime}}\right) .
$$

We can make a similar argument for players sharing the $i$ th highest bid, $i=3, \ldots, N$, if required. Therefore, no two players can share a bid for $\delta>0$ sufficiently low, and bids for adjacent positions must differ by exactly $\delta$. 
Equation (3) is Schaffer's (1988) equation (10), which exhibits not just the usual payoff maximization of the first term, but also a "spite" motivation in the second term (whose strength is increasing in the auction size $K$ relative to the population size $M$ ). Intuitively, a player's evolutionary success is dependent not on her raw payoff, but on her payoff relative to other players, with whom she is in ongoing competition. In the presence of this spite motivation, evolutionarily stable bids for adjacent positions must differ by $\delta$, otherwise, there would be an invasion by a mutant who bid a little more through spite; there would exist a player whose bid could be increased by $\delta$ at no cost to his own payoff but with resultant harm to another player. Without $\delta$ sufficiently low though, we would not be able to rule out multiple players sharing the same bid in an equilibrium ESS, which (as mentioned in Footnote 2) would make the position order $g$ stochastic and hence require a modified definition of locally envy-free equilibrium. Indeed, a player sharing a bid with a higher-value player would certainly "envy" that player in expectation (prior to the random resolution of their tie), violating one obvious modified concept of locally envy-free equilibrium.

I now show that, in the "playing the field" case with sufficiently low $\delta$, equilibrium ESS bids are locally envy-free. Intuitively, if positive equilibrium ESS bids differ by $\delta$, then an upwards mutation risks swapping positions with the bidder above and hence having to pay the higher bid-a scenario that is unprofitable by evolutionary stability, and which approaches the condition for locally envy-free equilibrium as $\delta$ becomes small. Similarly, if an equilibrium is not locally envy-free, then a bidder would like to swap bids with the bidder above her, which—if bids differ by $\delta$-allows an upwards mutation to destabilize the population as $\delta$ becomes small.

Proposition 2 There exists $\hat{\delta} \in(0, \bar{\delta}]$ such that, for all $\delta<\hat{\delta}$, if $K=M$ and $\sigma_{\text {ess }}$ is an equilibrium evolutionarily stable strategy, then the realized bids are locally envy-free.

Proof Given the evolutionarily stable $\sigma_{\text {ess }}$, consider a mutation $\sigma_{m}$ where, for some position $i \in\{2, \ldots, N\}$, bidder $g(i)$ bids $b_{\text {ess }}^{(i)}+\delta$ instead of $b_{\text {ess }}^{(i)}$, hence having a chance to swap positions with bidder $g(i-1)$ by Proposition 1 . If she were not the mutant, bidder $g(i)$ would get the position $i$ at a cost per click of $b_{\text {ess }}^{(i+1)}$, with payoff $\alpha_{i}\left(s_{g(i)}-b_{\mathrm{ess}}^{(i+1)}\right)$; although she faces an opponent playing the mutant strategy, this is irrelevant as she is the one in position $i$, and hence, each of her opponents plays $b_{\mathrm{ess}}^{(j)}$, $j \neq i$. Thus,

$$
\pi\left(\sigma_{\mathrm{ess}} \mid \sigma_{m}, \sigma_{\mathrm{ess}}, \ldots\right)=\frac{1}{K}\left(\alpha_{i}\left(s_{g(i)}-b_{\mathrm{ess}}^{(i+1)}\right)\right)+\beta
$$

for some $\beta>0$.

If she were the mutant, bidder $g(i)$ would have a chance of winning the position $i-1$ at a cost per click of $b_{\mathrm{ess}}^{(i)}+\delta$ with payoff $\alpha_{i-1}\left(s_{g(i)}-b_{\mathrm{ess}}^{(i)}-\delta\right)$. It follows that

$$
\pi\left(\sigma_{m} \mid \sigma_{\mathrm{ess}}, \sigma_{\mathrm{ess}}, \ldots\right)>\frac{1}{2 K}\left(\alpha_{i-1}\left(s_{g(i)}-b_{\mathrm{ess}}^{(i)}-\delta\right)+\alpha_{i}\left(s_{g(i)}-b_{\mathrm{ess}}^{(i+1)}\right)\right)+\beta
$$


where the strict inequality arises because $\sigma_{m}$ avoids the risk faced by $\sigma_{\text {ess }}$ of being outbid by the mutant for position $i-1$ when she is bidder $g(i-1)$, so that her expected payoff in positions other than $i$ exceeds $\beta$.

If $K=M$, it follows by (2) that

$$
\alpha_{i}\left(s_{g(i)}-b_{\mathrm{ess}}^{(i+1)}\right)>\alpha_{i-1}\left(s_{g(i)}-b_{\mathrm{ess}}^{(i)}-\delta\right),
$$

from which the result follows for $\delta$ sufficiently low.

In other words, in the "playing the field" case where the whole population participates in each auction, an equilibrium strategy that is evolutionarily stable must produce locally envy-free bids for sufficiently low $\delta$. If a proper subset of the population plays each auction, then the bidders' "spite" motivation is diluted; when $K<M$, the weighting on the " $-\pi\left(\sigma_{\text {ess }} \mid \sigma_{m}, \sigma_{\text {ess }}, \ldots\right)$ " term of (3) is reduced from 1 to $(K-$ $1) /(M-1)$, and as a result (2) no longer implies (4). In other words, outside of the "playing the field" case, I cannot say whether evolutionarily stable bids are locally envy-free or not.

However, the converse result to Proposition 2 does not hold; a locally envy-free equilibrium need not be evolutionarily stable, as the following example shows for the case of the popular VCG outcome.

Example 1 Suppose that $N=1, K=M=2$ and $S=\{2,1\}$, giving a second-price auction. Let $\sigma \equiv(\sigma(2), \sigma(1))=(b+\delta, b)$, and consider two possible mutations $\sigma^{\prime} \equiv\left(\sigma^{\prime}(2), \sigma^{\prime}(1)\right)=(b, b)$, and $\sigma^{\prime \prime} \equiv\left(\sigma^{\prime \prime}(2), \sigma^{\prime \prime}(1)\right)=(b+\delta, b+\delta)$. Then a bidder with strategy $\sigma$ has: value 2 with probability $1 / 2$, in which case she bids $b+\delta$ and beats the $b$ bid of strategy $\sigma^{\prime}$; and value 1 with probability $1 / 2$, in which case she ties the $b$ bid of strategy $\sigma^{\prime}$ and receives the position with conditional probability $1 / 2$. Thus,

$$
\begin{aligned}
\pi\left(\sigma \mid \sigma^{\prime}\right)=\frac{1}{2} \alpha_{1}(2-b)+\frac{1}{4} \alpha_{1}(1-b) & \geq \frac{1}{4} \alpha_{1}(2-b)=\pi\left(\sigma^{\prime} \mid \sigma\right) \\
\Leftrightarrow \quad b & \leq 3 / 2
\end{aligned}
$$

and similarly,

$$
\begin{aligned}
\pi\left(\sigma \mid \sigma^{\prime \prime}\right)=\frac{1}{4} \alpha_{1}(2-b-\delta) & \geq \frac{1}{2} \alpha_{1}(2-b)+\frac{1}{4} \alpha_{1}(1-b-\delta)=\pi\left(\sigma^{\prime \prime} \mid \sigma\right) \\
\Leftrightarrow \quad b & \geq 3 / 2 .
\end{aligned}
$$

Since these are the most attractive mutations (by Lemma 1), $\sigma$ is evolutionarily stable for $b=3 / 2$, in which case it is also an equilibrium strategy and locally envy-free. For general $S$, the stable bid is $b=\left(s_{1}+s_{2}\right) / 2$. Note, however, that the VCG outcome is given by strategies of the form $\sigma_{\mathrm{VCG}} \equiv\left(\sigma_{\mathrm{VCG}}(2), \sigma_{\mathrm{VCG}}(1)\right)=(b, 1), b>1$; only $(1+\delta, 1)$ is a candidate for evolutionary stability when $\delta$ is low enough to satisfy Proposition 1, and it is vulnerable to invasion by $\sigma^{\prime \prime}$. Thus, the VCG outcome need not be evolutionarily stable. 


\subsection{Existence}

But the existence of an evolutionarily stable strategy is not guaranteed in general, and indeed, deciding its existence in the infinite-population case is both NP-hard and coNP-hard (Etessami and Lochbiler 2005). Moreover, even if existence of ESS does hold for a particular GSP, it is not clear that there exists an ESS that is also an equilibrium strategy. Here I establish that such an equilibrium ESS does exist for the "playing the field" GSP with one position, and also for a large number of bidders on two positions (which I then argue extends to more than two positions). But first I establish a useful result that drastically reduces the number of mutations that need to be considered.

Lemma 1 Given an equilibrium strategy $\sigma_{\mathrm{ess}}$, if $\delta$ is sufficiently low and the evolutionary stability conditions (2) hold for:

- mutations where a bidder $k \in\{2, \ldots, N+1\}$ switches to the bid $b_{k-1}$; and

- mutations where a bidder $k \in\{1, \ldots, N\}$ switches to the bid $b_{k+1}$;

then they hold for all mutations.

Proof Consider a mutation $\sigma_{m}^{\prime}$ that has a bidder $k$ switch her bid to any $b_{g(i)} \in$ $\left\{b_{1}, \ldots, b_{k-2}\right\}$ (i.e. switching to a nonadjacent higher position $i$ ), and let $\sigma_{m}$ be the mutation where bidder $k-1$ (rather than bidder $k$ ) switches to $b_{g(i)}$. Then $\pi\left(\sigma_{m} \mid \sigma_{\mathrm{ess}}, \sigma_{\mathrm{ess}}, \ldots\right)>\pi\left(\sigma_{m}^{\prime} \mid \sigma_{\mathrm{ess}}, \sigma_{\mathrm{ess}}, \ldots\right)$, since $s_{k-1}>s_{k}$ and bidder $k-1$ suffers a smaller increase in her expected payment than bidder $k$. Moreover, $\pi\left(\sigma_{\mathrm{ess}} \mid \sigma_{m}^{\prime}, \sigma_{\mathrm{ess}}, \ldots\right)>\pi\left(\sigma_{\mathrm{ess}} \mid \sigma_{m}, \sigma_{\mathrm{ess}}, \ldots\right)$, since bidder $k$ lowers the payment of bidder $k-1$ under $\sigma_{m}^{\prime}$ (thus gaining a lower "spite" benefit than bidder $k-1$ from the deviation). It follows that, if (2) holds for $\sigma_{m}$, then it also holds for $\sigma_{m}^{\prime}$. We may then proceed by induction until we reach bidder $g(i+1)$.

Consider a mutation $\sigma_{m}^{\prime}$ that has a bidder $k$ switch her bid to any $b_{g(i)} \in$ $\left\{b_{k+2}, b_{k+3}, \ldots, b_{N}\right\}$ (i.e. switching to a nonadjacent lower position $i$ ), and let $\sigma_{m}$ be the mutation where bidder $k+1$ (rather than bidder $k$ ) switches to $b_{g(i)}$. Then $\pi\left(\sigma_{m} \mid \sigma_{\mathrm{ess}}, \sigma_{\mathrm{ess}}, \ldots\right)>\pi\left(\sigma_{m}^{\prime} \mid \sigma_{\mathrm{ess}}, \sigma_{\mathrm{ess}}, \ldots\right)$ for sufficiently low $\delta$, since $s_{k+1}<s_{k}$ and bidder $k$ 's expected payment under $\sigma_{m}^{\prime}$ falls by only $\delta$ more than $k+1$ 's expected payment under $\sigma_{m}$ (by Proposition 1). Moreover, $\pi\left(\sigma_{\mathrm{ess}} \mid \sigma_{m}^{\prime}, \sigma_{\mathrm{ess}}, \ldots\right)=$ $\pi\left(\sigma_{\mathrm{ess}} \mid \sigma_{m}, \sigma_{\mathrm{ess}}, \ldots\right)$, since bidder $k$ lowers the payment of bidder $k-1$ by $\delta$ under $\sigma_{m}^{\prime}$, bidder $k+1$ lowers the payment of bidder $k$ by $\delta$ under $\sigma_{m}$, and other bidders are affected the same by the two mutations. It follows that, if $\delta$ is sufficiently low and (2) holds for $\sigma_{m}$, then it also holds for $\sigma_{m}^{\prime}$. We may then proceed by induction until we reach bidder $g(i-1)$.

Finally, if a mutation $\sigma_{m}$ has a bidder $k \in\{N+1, N+2, \ldots\}$ switch her bid to any $b \in\left\{b_{N+2}, b_{N+3}, \ldots, b_{K}\right\}$, then the bidder neither wins a position nor affects any other bidder's payment, under either $\sigma_{\mathrm{ess}}$ or $\sigma_{m}$; hence, $\pi\left(\sigma_{\mathrm{ess}} \mid \sigma_{m}, \sigma_{\mathrm{ess}}, \ldots\right)=$ $\pi\left(\sigma_{\text {ess }} \mid \sigma_{\text {ess }}, \sigma_{\text {ess }}, \ldots\right)$. Moreover, since $\sigma_{\text {ess }}$ is an equilibrium strategy, we have $\pi\left(\sigma_{\mathrm{ess}} \mid \sigma_{\mathrm{ess}}, \sigma_{\mathrm{ess}}, \ldots\right) \geq \pi\left(\sigma_{m} \mid \sigma_{\mathrm{ess}}, \sigma_{\mathrm{ess}}, \ldots\right)$, and condition (2) holds.

Thus, we need only consider mutations where a subset of bidders switch to an adjacent position, similar to the sufficiency of adjacent deviations for symmetric equilibrium 
(Varian 2007, Fact 5). Note that it follows that every equilibrium local ESS (AlósFerrer and Ania 2001) is an ESS in the GSP. Henceforth, I suppose that $\delta$ is low enough to satisfy the conditions of Propositions 1, 2 and Lemma 1.

With Lemma 1 in hand, it becomes easier to check for existence of an equilibrium ESS, as in the following two results.

Proposition 3 Suppose that $N=1$ and $K=M \geq 2$, and consider the strategy $\sigma \equiv\left(\sigma\left(s_{1}\right), \sigma\left(s_{2}\right), \sigma\left(s_{3}\right), \sigma\left(s_{4}\right), \ldots\right)=(b+\delta, b, 0,0, \ldots)$. Then $\sigma$ is an equilibrium strategy and evolutionarily stable for

$$
b \in\left[\frac{1}{K} s_{1}+\frac{K-1}{K} s_{2}-\frac{K-2}{K} \delta, \frac{K-1}{K} s_{1}+\frac{1}{K} s_{2}\right] \neq \emptyset .
$$

Proof Consider two possible mutations, $\sigma^{\prime} \equiv\left(\sigma^{\prime}\left(s_{1}\right), \sigma^{\prime}\left(s_{2}\right), \sigma^{\prime}\left(s_{3}\right), \sigma^{\prime}\left(s_{4}\right), \ldots\right)=$ $(b, b, 0,0, \ldots)$ and $\sigma^{\prime \prime} \equiv\left(\sigma^{\prime \prime}\left(s_{1}\right), \sigma^{\prime \prime}\left(s_{2}\right), \sigma^{\prime \prime}\left(s_{3}\right), \sigma^{\prime \prime}\left(s_{4}\right), \ldots\right)=(b+\delta, b+$ $\delta, 0,0, \ldots)$. Then

$$
\begin{aligned}
\pi\left(\sigma \mid \sigma^{\prime}, \sigma, \ldots\right) & =\frac{1}{K} \alpha_{1}\left(s_{1}-b\right)+\frac{1}{2 K(K-1)} \alpha_{1}\left(s_{2}-b\right) \\
& \geq \frac{1}{2 K} \alpha_{1}\left(s_{1}-b\right)=\pi\left(\sigma^{\prime} \mid \sigma, \sigma, \ldots\right) \\
\Leftrightarrow \quad b & \leq \frac{(K-1) s_{1}+s_{2}}{K}
\end{aligned}
$$

and

$$
\begin{aligned}
\pi\left(\sigma \mid \sigma^{\prime \prime}, \sigma, \ldots\right) & =\frac{1}{2 K(K-1)} \alpha_{1}\left(s_{1}-b-\delta\right)+\frac{K-2}{K(K-1)} \alpha_{1}\left(s_{1}-b\right) \\
& \geq \frac{1}{K} \alpha_{1}\left(s_{1}-b\right)+\frac{1}{2 K} \alpha_{1}\left(s_{2}-b-\delta\right)=\pi\left(\sigma^{\prime \prime} \mid \sigma, \sigma, \ldots\right) \\
\Leftrightarrow \quad b & \geq \frac{s_{1}+(K-1) s_{2}}{K}-\frac{K-2}{K} \delta .
\end{aligned}
$$

By Lemma 1, $\sigma$ is evolutionarily stable for such a $b$. And since $\sigma\left(s_{2}\right)=b<s_{1}$ and $\sigma\left(s_{1}\right)=b+\delta>s_{2}, \sigma$ is also an equilibrium strategy.

Notice that, as $K \rightarrow \infty$ and $\delta \rightarrow 0, \sigma$ becomes evolutionarily stable for all $b$ in the interval $\left(s_{2}, s_{1}\right)$.

Proposition 4 Suppose that $N=2$ and $K=M$, and consider the strategy $\sigma \equiv$ $\left(\sigma\left(s_{1}\right), \sigma\left(s_{2}\right), \sigma\left(s_{3}\right), \sigma\left(s_{4}\right), \ldots\right)=(b+\delta, b, b-\delta, 0, \ldots)$. Then, as $K=M \rightarrow \infty$, $\sigma$ is an equilibrium strategy and evolutionarily stable for

$$
b \in\left[s_{2}-\frac{\alpha_{1}+\alpha_{2}}{\alpha_{1}-\alpha_{2}} \delta, s_{2}+\delta\right] .
$$

Proof Consider four possible mutations, 


$$
\begin{aligned}
\sigma^{\prime} & \equiv\left(\sigma^{\prime}\left(s_{1}\right), \sigma^{\prime}\left(s_{2}\right), \sigma^{\prime}\left(s_{3}\right), \sigma^{\prime}\left(s_{4}\right), \ldots\right)=(b, b, b-\delta, 0, \ldots), \\
\sigma^{\prime \prime} & \equiv\left(\sigma^{\prime \prime}\left(s_{1}\right), \sigma^{\prime \prime}\left(s_{2}\right), \sigma^{\prime \prime}\left(s_{3}\right), \sigma^{\prime \prime}\left(s_{4}\right), \ldots\right)=(b+\delta, b+\delta, b-\delta, 0, \ldots), \\
\sigma^{\prime \prime \prime} & \equiv\left(\sigma^{\prime \prime \prime}\left(s_{1}\right), \sigma^{\prime \prime \prime}\left(s_{2}\right), \sigma^{\prime \prime \prime}\left(s_{3}\right), \sigma^{\prime \prime \prime}\left(s_{4}\right), \ldots\right)=(b+\delta, b-\delta, b-\delta, 0, \ldots), \quad \text { and } \\
\sigma^{\prime \prime \prime \prime \prime} & \equiv\left(\sigma^{\prime \prime \prime \prime \prime}\left(s_{1}\right), \sigma^{\prime \prime \prime \prime}\left(s_{2}\right), \sigma^{\prime \prime \prime \prime}\left(s_{3}\right), \sigma^{\prime \prime \prime \prime}\left(s_{4}\right), \ldots\right)=(b+\delta, b, b, 0, \ldots) .
\end{aligned}
$$

Then

$$
\begin{aligned}
& \pi\left(\sigma \mid \sigma^{\prime}, \sigma, \ldots\right)=\frac{1}{K} \alpha_{1}\left(s_{1}-b\right)+\frac{1}{2 K(K-1)}\left(\alpha_{1}\left(s_{2}-b\right)+\alpha_{2}\left(s_{2}-b+\delta\right)\right) \\
& +\frac{K-2}{K(K-1)} \alpha_{2}\left(s_{2}-b+\delta\right) \\
& \geq \frac{1}{2 K}\left(\alpha_{1}\left(s_{1}-b\right)+\alpha_{2}\left(s_{1}-b+\delta\right)\right) \\
& +\frac{1}{K} \alpha_{2}\left(s_{2}-b+\delta\right)=\pi\left(\sigma^{\prime} \mid \sigma, \sigma, \ldots\right) \\
& \Leftrightarrow \quad b \leq \frac{(K-1) s_{1}+s_{2}}{K}-\frac{\alpha_{2}}{\alpha_{1}-\alpha_{2}} \delta, \\
& \pi\left(\sigma \mid \sigma^{\prime \prime}, \sigma, \ldots\right)=\frac{1}{2 K(K-1)}\left(\alpha_{1}\left(s_{1}-b-\delta\right)+\alpha_{2}\left(s_{1}-b+\delta\right)\right) \\
& +\frac{K-2}{K(K-1)} \alpha_{1}\left(s_{1}-b\right)+\frac{1}{K} \alpha_{2}\left(s_{2}-b+\delta\right) \\
& \geq \frac{1}{K} \alpha_{1}\left(s_{1}-b\right) \\
& +\frac{1}{2 K}\left(\alpha_{1}\left(s_{2}-b-\delta\right)+\alpha_{2}\left(s_{2}-b+\delta\right)\right)=\pi\left(\sigma^{\prime \prime} \mid \sigma, \sigma, \ldots\right) \\
& \Leftrightarrow \quad b \geq \frac{s_{1}+(K-1) s_{2}}{K}-\frac{(K-2) \alpha_{1}+K \alpha_{2}}{K\left(\alpha_{1}-\alpha_{2}\right)} \delta, \\
& \pi\left(\sigma \mid \sigma^{\prime \prime \prime}, \sigma, \ldots\right)=\frac{1}{K(K-1)}\left(\alpha_{1}\left(s_{1}-b+\delta\right)+\frac{\alpha_{2}}{2}\left(s_{3}-b+\delta\right)\right) \\
& +\frac{K-2}{K(K-1)} \alpha_{1}\left(s_{1}-b\right)+\frac{1}{K} \alpha_{2}\left(s_{2}-b+\delta\right) \\
& \geq \frac{1}{K} \alpha_{1}\left(s_{1}-b\right)+\frac{1}{2 K} \alpha_{2}\left(s_{2}-b+\delta\right)=\pi\left(\sigma^{\prime \prime \prime} \mid \sigma, \sigma, \ldots\right) \\
& \Leftrightarrow \quad b \leq \frac{(K-1) s_{2}+s_{3}}{K}+\frac{2 \alpha_{1}+K \alpha_{2}}{K \alpha_{2}} \delta,
\end{aligned}
$$

and

$$
\begin{aligned}
\pi\left(\sigma \mid \sigma^{\prime \prime \prime \prime}, \sigma, \ldots\right)= & \frac{1}{K} \alpha_{1}\left(s_{1}-b\right)+\frac{1}{2 K(K-1)} \alpha_{2}\left(s_{2}-b\right) \\
& +\frac{K-2}{K(K-1)} \alpha_{2}\left(s_{2}-b+\delta\right) \\
& \geq \frac{1}{K} \alpha_{1}\left(s_{1}-b\right)+\frac{1}{K} \alpha_{2}\left(s_{2}-b+\delta\right)
\end{aligned}
$$




$$
\begin{gathered}
+\frac{1}{2 K} \alpha_{2}\left(s_{3}-b\right)=\pi\left(\sigma^{\prime \prime \prime \prime} \mid \sigma, \sigma, \ldots\right) \\
\Leftrightarrow \quad b \geq \frac{s_{2}+(K-1) s_{3}}{K}+\frac{2}{K} \delta .
\end{gathered}
$$

By Lemma 1, these are the conditions for $\sigma$ 's evolutionary stability, satisfied for

$$
\begin{gathered}
b \in\left[\max \left\{\frac{1}{K} s_{1}+\frac{K-1}{K} s_{2}-\frac{K\left(\alpha_{1}+\alpha_{2}\right)-2 \alpha_{1}}{K\left(\alpha_{1}-\alpha_{2}\right)} \delta, \frac{1}{K} s_{2}+\frac{K-1}{K} s_{3}+\frac{2}{K} \delta\right\},\right. \\
\left.\min \left\{\frac{K-1}{K} s_{1}+\frac{1}{K} s_{2}-\frac{\alpha_{2}}{\alpha_{1}-\alpha_{2}} \delta, \frac{K-1}{K} s_{2}+\frac{1}{K} s_{3}+\frac{2 \alpha_{1}+K \alpha_{2}}{K \alpha_{2}} \delta\right\}\right],
\end{gathered}
$$

which (for low $\delta$ ) approaches the interval $\left[s_{2}-\delta\left(\alpha_{1}+\alpha_{2}\right) /\left(\alpha_{1}-\alpha_{2}\right), s_{2}+\delta\right]$ as $K \rightarrow \infty$.

Furthermore, $\sigma$ is an equilibrium strategy if:

$$
\begin{aligned}
\alpha_{1}\left(s_{1}-\sigma\left(s_{2}\right)\right) & \geq \frac{1}{2}\left(\alpha_{1}\left(s_{1}-\sigma\left(s_{2}\right)\right)+\alpha_{2}\left(s_{1}-\sigma\left(s_{3}\right)\right)\right) \\
\Leftrightarrow \quad \alpha_{1}\left(s_{1}-b\right) & \geq \alpha_{2}\left(s_{1}-b+\delta\right) \\
\Leftrightarrow \quad b & \leq s_{1}-\frac{\alpha_{2}}{\alpha_{1}-\alpha_{2}} \delta,
\end{aligned}
$$

which is implied by (5); and if:

$$
\begin{aligned}
\alpha_{2}\left(s_{2}-\sigma\left(s_{3}\right)\right) & \geq \frac{1}{2}\left(\alpha_{1}\left(s_{2}-\sigma\left(s_{1}\right)\right)+\alpha_{2}\left(s_{2}-\sigma\left(s_{3}\right)\right)\right) \\
\Leftrightarrow \quad \alpha_{2}\left(s_{2}-b+\delta\right) & \geq \alpha_{1}\left(s_{2}-b-\delta\right) \\
\Leftrightarrow \quad b & \geq s_{2}-\frac{\alpha_{1}+\alpha_{2}}{\alpha_{1}-\alpha_{2}} \delta \\
\Leftrightarrow \quad \alpha_{2}\left(s_{2}-b+\delta\right) & \geq 0 \\
\Leftrightarrow \quad b & \leq s_{2}+\delta ; \text { and } \\
\alpha_{2}\left(s_{2}-\sigma\left(s_{3}\right)\right) & \geq \frac{1}{2} \alpha_{2}\left(s_{2}-\sigma\left(s_{3}\right)\right) \\
\Leftrightarrow \quad 0 & \geq \frac{1}{2} \alpha_{2}\left(s_{3}-\sigma\left(s_{2}\right)\right) \\
\Leftrightarrow \quad 0 & \geq \alpha_{2}\left(s_{3}-b\right) \\
\Leftrightarrow \quad b & \geq s_{3}
\end{aligned}
$$

the latter of which is implied by (6).

By contrast with Proposition 3, this existence result required an arbitrarily large number of bidders, $K=M \rightarrow \infty$, in order to guarantee stability against the larger number of mutations possible under two positions. Whilst the population size thus returns to infinity, the "playing the field" assumption $K=M$ is maintained, and hence so are Schaffer's (1988) finite-population ESS effects. Intuitively, as we take 
this limit, the chance of being in an adjacent position to the single mutant vanishes, so that the "playing the field" evolutionary stability conditions,

$$
\pi\left(\sigma_{\mathrm{ess}} \mid \sigma_{m}, \sigma_{\mathrm{ess}}, \ldots\right) \geq \pi\left(\sigma_{m} \mid \sigma_{\mathrm{ess}}, \sigma_{\mathrm{ess}}, \ldots\right), \quad \forall \sigma_{m},
$$

approach the conditions for an equilibrium strategy,

$$
\pi\left(\sigma_{\mathrm{ess}} \mid \sigma_{\mathrm{ess}}, \sigma_{\mathrm{ess}}, \ldots\right) \geq \pi\left(\sigma_{m} \mid \sigma_{\mathrm{ess}}, \sigma_{\mathrm{ess}}, \ldots\right), \quad \forall \sigma_{m}
$$

For this reason — and by existence of Nash equilibrium—-similar existence results will hold with more than two positions, although of course direct verification becomes ever more cumbersome as $N$ (and hence the number of mutations to be checked) grows.

\section{Conclusion}

"Locally envy-free" (or "symmetric") equilibrium has been a very influential concept in the analysis of the generalized second-price auction, but its theoretical foundations have also been criticized. Why should a bidder wish to increase her bid to harm her opponent, and why should she cease to do so in case her bid is undercut by the bidder in the position above? Evolutionary stability provides an answer to both of these questions, and it does so under the "playing the field" assumption that is most consistent with the standard complete-information approach to the GSP. An evolutionarily stable bidder is "spiteful" towards her opponents, intuitively because she is in ongoing competition with them for survival, and their loss is hence her gain. For this reason, positive evolutionarily stable bids are consecutive, and any upwards mutation would tie the bid in the position above; an evolutionarily stable bidder must be able to outperform such a mutation, just as she must be able to outperform all manner of "mutant" strategies. Outside of the "playing the field" case, the evolutionary foundation for locally envy-free equilibrium may or may not hold, but with a fixed group of bidders, evolutionary stability and locally envy-free equilibrium go hand-in-hand.

Open Access This article is licensed under a Creative Commons Attribution 4.0 International License, which permits use, sharing, adaptation, distribution and reproduction in any medium or format, as long as you give appropriate credit to the original author(s) and the source, provide a link to the Creative Commons licence, and indicate if changes were made. The images or other third party material in this article are included in the article's Creative Commons licence, unless indicated otherwise in a credit line to the material. If material is not included in the article's Creative Commons licence and your intended use is not permitted by statutory regulation or exceeds the permitted use, you will need to obtain permission directly from the copyright holder. To view a copy of this licence, visit http://creativecommons.org/licenses/by/4.0/.

\section{References}

Alós-Ferrer, C., Ania, A.B.: Local equilibria in economic games. Econ. Lett. 70, 165-173 (2001)

Alós-Ferrer, C., Ania, A.B.: The evolutionary stability of perfectly competitive behavior. Econ. Theory 26, 497-516 (2005). https://doi.org/10.1007/s00199-004-0474-8

Andreoni, J., Che, Y., Kim, J.: Asymmetric information about rivals' types in standard auctions: an experiment. Games Econ. Behav. 59, 240-259 (2007) 
Ania, A.B.: Evolutionary stability and nash equilibrium in finite populations, with an application to price competition. J. Econ. Behav. Org. 65, 472-488 (2008)

Athey, S., Ellison, G.: Position auctions with consumer search. Q. J. Econ. 126, 1213-1270 (2011)

Athey, S., Nekipelov, D.: A structural model of sponsored search advertising auctions. Working paper, Microsoft Research (2010)

Börgers, T., et al.: Equilibrium bids in sponsored search auctions: theory and evidence. Am. Econ. J. Microecon. 5, 163-187 (2013)

Caragiannis, I., et al.: Bounding the inefficiency of outcomes in generalized second price auctions. J. Econ. Theory 156, 343-388 (2015). https://doi.org/10.1016/j.jet.2014.04.010

Cary, M., et al.: Convergence of position auctions under myopic best-response dynamics. ACM Trans. Econ. Comput. 2, 1-20 (2014)

Che, Y.-K., Choi, S., Kim, J.: An experimental study of sponsored-search auctions. Games Econ. Behav. 102, 20-43 (2017)

Cooper, D.J., Fang, H.: Understanding overbidding in second price auctions: an experimental study. Econ. J. 118, 1572-1595 (2008)

Decarolis, F., Goldmanis, M., Penta, A.: Marketing agencies and collusive bidding in online ad auctions. Working paper (2017)

Edelman, B., Ostrovsky, M., Schwartz, M.: Internet advertising and the generalized second-price auction: selling billions of dollars worth of keywords. Am. Econ. Rev. 97, 242-259 (2007)

Etessami, K., Lochbiler, A.: The computational complexity of evolutionarily stable strategies. Electronic Colloquium on Computational Complexity (ECCC) (055) (2005)

Gomes, R., Sweeney, K.: Bayes-Nash equilibria of the generalized second-price auction. Games Econ. Behav. 86, 421-437 (2014)

Hamilton, W.D.: Selection of selfish and altruistic behavior in some extreme models. In: Eisenberg, J.F., Dillon, W.S. (eds.) Chapter 6 in Man and Beast: Comparative Social Behavior. Smithsonian Institution, Washington, D.C. (1971)

Hashimoto, T.: Equilibrium selection, inefficiency, and instability in internet advertising auctions. Working paper (2013)

Hehenkamp, B., Possajennikov, A., Guse, T.: On the equivalence of Nash and evolutionary equilibrium in finite populations. J. Econ. Behav. Org. 73, 254-258 (2010)

Jordan, J.S.: Bayesian learning in normal form games. Games Econ. Behav. 3, 60-81 (1991)

Kohlberg, E., Mertens, J.-F.: On the strategic stability of equilibria. Econometrica 54, 1003-1037 (1986)

Maynard Smith, J.: Evolution and the Theory of Games. Cambridge University Press, Cambridge (1982)

Maynard Smith, J., Price, G.R.: The logic of animal conflict. Nature 246, 15-18 (1973)

Milgrom, P., Mollner, J.: Extended proper equilibrium. Working paper. (2018b)

Milgrom, P., Mollner, J.: Equilibrium selection in auctions and high stakes games. Econometrica 86, 219261 (2018a)

Morgan, J., Steiglitz, K., Reis, G.: The spite motive and equilibrium behavior in auctions. Contrib. Econ. Anal. Policy 2, 5 (2003)

Myerson, R.B.: Refinements of the Nash equilibrium concept. Int. J. Game Theory 7, 73-80 (1978)

Newton, J.: Evolutionary game theory: a renaissance. Games 9, 31 (2018)

Nishimura, N., et al.: Spite andreciprocity in auctions. Games 2, 365-411 (2011)

Sandholm, W.H.: Population Games and Evolutionary Dynamics. The MIT Press, Cambridge (2010)

Schaffer, M.E.: Evolutionarily stable strategies for a finite population and a variable contest size. J. Theor. Biol. 132, 469-478 (1988)

Selten, R.: A note on evolutionarily stable strategies in asymmetric animal contests. J. Theor. Biol. 84, 93-101 (1980)

van Damme, E.: A relation between perfect equilibria in extensive form games and proper equilibria in normal form games. Int. J. Game Theory 13, 1-13 (1984)

Varian, H.R.: Position auctions. Int. J. Ind. Org. 25, 1163-1178 (2007)

Vega-Redondo, F.: Evolution, Games and Economic Behavior. Oxford University Press, Oxford (1996)

Vega-Redondo, F.: The evolution of Walrasian behavior. Econometrica 65, 375-384 (1997)

Weibull, J.W.: Evolutionary Game Theory. The MIT Press, Cambridge (1995)

Publisher's Note Springer Nature remains neutral with regard to jurisdictional claims in published maps and institutional affiliations. 